University of Nebraska - Lincoln

DigitalCommons@University of Nebraska - Lincoln

$7-24-2000$

\title{
Surface electronic phase transition in colossal magnetoresistive manganese perovskites: La0.65 $\mathrm{Sr}_{0.35} \mathrm{MnO}_{3}$
}

\author{
Hani Dulli \\ University of Tennessee, Knoxville, hani.dulli@uconn.edu \\ E. Ward Plummer \\ University of Tennessee, Knoxville,wplummer@phys.Isu.edu \\ Peter A. Dowben \\ University of Nebraska-Lincoln, pdowben@unl.edu \\ Jaewu Choi \\ University of Nebraska-Lincoln, jchoi@ece.eng.wayne.edu \\ Sy_Hwang Liou \\ University of Nebraska-Lincoln, sliou@unl.edu
}

Follow this and additional works at: https://digitalcommons.unl.edu/physicsdowben

Part of the Physics Commons

Dulli, Hani; Plummer, E. Ward; Dowben, Peter A.; Choi, Jaewu; and Liou, Sy_Hwang, "Surface electronic phase transition in colossal magnetoresistive manganese perovskites: La0.65Sr0.35 $\mathrm{MnO}_{3}$ " (2000). Peter Dowben Publications. 28.

https://digitalcommons.unl.edu/physicsdowben/28

This Article is brought to you for free and open access by the Research Papers in Physics and Astronomy at DigitalCommons@University of Nebraska - Lincoln. It has been accepted for inclusion in Peter Dowben Publications by an authorized administrator of DigitalCommons@University of Nebraska - Lincoln. 


\title{
Surface electronic phase transition in colossal magnetoresistive manganese perovskites: $\mathrm{La}_{0.65} \mathrm{Sr}_{0.35} \mathrm{MnO}_{3}$
}

\author{
Hani Dulli and E. W. Plummera) \\ Department of Physics and Astronomy, University of Tennessee, Knoxville, Tennessee 37996 \\ and Solid State Division, Oak Ridge National Laboratory, Oak Ridge, Tennessee 37831 \\ P. A. Dowben, Jaewu Choi, and S.-H. Liou \\ Department of Physics and Astronomy and the Center for Materials Research and Analysis, \\ Behlen Laboratory of Physics, University of Nebraska, Lincoln, Nebraska 68588
}

(Received 22 March 2000; accepted for publication 25 May 2000)

\begin{abstract}
We have observed a distinct surface phase transition for an important class of giant magnetoresistance materials $\left[\mathrm{La}_{1-x} \mathrm{Sr}_{x} \mathrm{MnO}_{3}(x=0.35)\right]$. The surface phase transition occurs at 240 $\mathrm{K}$ compared to $370 \mathrm{~K}$ for the bulk and is fundamentally different. In the bulk, a ferromagnetic metal to paramagnetic bad-metal transition occurs, while the lower-temperature surface transition is from an insulator to a semimetal. The surface of this manganese perovskite is electronically and compositionally quite different from the bulk with important implications for the behavior of artificially grown layered transition-metal oxides and for the use of surface sensitive techniques to probe the bulk. (c) 2000 American Institute of Physics. [S0003-6951(00)00630-6]
\end{abstract}

The creation of a surface breaks the symmetry of a crystal inducing electronic, magnetic, and geometrical changes, which will change the order parameters associated with various types of phase transitions. Thus with strong coupling among the spin, charge, and lattice degrees of freedom in transition metal oxides (TMOs), ${ }^{1}$ dramatic changes in the order parameters should be expected at the surface. Furthermore, the surface free energy should be different from the bulk free energy inducing surface segregation. ${ }^{2}$ Since the phase diagram of TMOs is critically dependent upon the doping concentration, the surface could be a "different material." In addition, many of the surfaces in the TMOs will be polar surfaces, i.e., the net charge in the plane is not zero. This too will drive either a structural or electronic reconstruction at the surface. Despite the fascinating possibilities for observing new surface phases and the technological potential of artificially structured TMO layered materials, ${ }^{3}$ there have been surprisingly few studies of the properties of their surfaces or interfaces. Understanding the surface phases of these materials will also benefit interpretation of surface sensitive experimental techniques such as angle resolved photoemission.

We have observed a distinct surface phase transition for the TMO La $\mathrm{La}_{0.6} \mathrm{Sr}_{0.35} \mathrm{MnO}_{3}$. At a temperature of $240 \mathrm{~K}$ the surface undergoes an insulator to semimetal phase transition, while bulk measurements on the same sample show a metallic-ferromagnetic to semiconductor (bad-metal) paramagnetic transition at $370 \mathrm{~K}$. There have been indications of the low temperature surface insulating phase in other studies on spin-polarized tunneling ${ }^{4}$ and thickness-dependent magnetotransport ${ }^{5}$ in $\mathrm{La}_{0.67} \mathrm{Sr}_{0.33} \mathrm{MnO}_{3}$ thin films.

$\mathrm{La}_{1-x} \mathrm{Sr}_{x} \mathrm{MnO}_{3}(x=0.35)$ thin films (nominal thickness of $1000 \AA$ ) were grown on (100) $\mathrm{LaAlO}_{3}$ substrates by rf sputtering in a 2:1 argon/oxygen atmosphere maintained at

${ }^{a)}$ Electronic mail: eplummer@utk.edu
20 mTorr. The bulk chemical composition of the films was determined from energy dispersive analysis of x-ray emission spectroscopy and found to be similar to the targets with the final compositions $\mathrm{La}_{0.65} \mathrm{Sr}_{0.35} \mathrm{MnO}_{3}$. The films were subsequently annealed at $900{ }^{\circ} \mathrm{C}$ in an oxygen atmosphere of 2 atm for $10 \mathrm{~h}$. The crystallinity and orientation was established by x-ray diffraction and the electronic and magnetic properties were determined with temperature dependent resistivity and magnetization measurements. Appropriate surface preparation was established by $\mathrm{x}$-ray photoemission spectroscopy (XPS), ultraviolet photoemission, and inverse photoemission. Prior to each experiment, samples were cleaned by annealing at $500{ }^{\circ} \mathrm{C}$ for $2 \mathrm{~h}$. XPS measurements were undertaken with the $\operatorname{Mg} K \alpha$ line $(1253.6 \mathrm{eV})$ using a commercial system. The angle resolved inverse photoemission spectra were obtained on a home made system by using variable energy electrons and a Geiger-Müller detector. In our scheme, $9.8 \mathrm{eV}$ photons were detected with the GeigerMüller tube with $\sim 400 \mathrm{meV}$ bandwidth. The overall energy resolution in inverse photoemission was $\sim 450 \mathrm{meV}$.

Figure 1 compares our measurements of the bulk (a) and surface (b) properties of a $\sim 1000 \AA$ thick film of $\mathrm{La}_{0.65} \mathrm{Sr}_{0.35} \mathrm{MnO}_{3}$. Panel (a) shows the magnetization and the resistivity indicating that the transition is at $T_{C}^{B} \sim 370 \mathrm{~K}$, consistent with measurement of bulk samples. ${ }^{1}$ In panel (b) the surface sensitive measurements are displayed. The solid circles represent the $\mathrm{O} 1 s$ core level binding energy as a function of temperature. The binding energies of the core levels shift to greater binding energies with decreasing temperature, where the extent of the shift is observed to strongly depend upon the x-ray source power (flux). The greater the incident x-ray flux becomes, the larger is the core level shift (toward higher binding energies) for temperatures below 240 $\mathrm{K}$. The fact that the core level binding energies depend upon $\mathrm{x}$-ray flux indicated that surface charging of the sample occurs below $240 \mathrm{~K}$. The open circles represent the density of states at the Fermi energy (integrated from -0.3 to $+0.3 \mathrm{eV}$ ) 


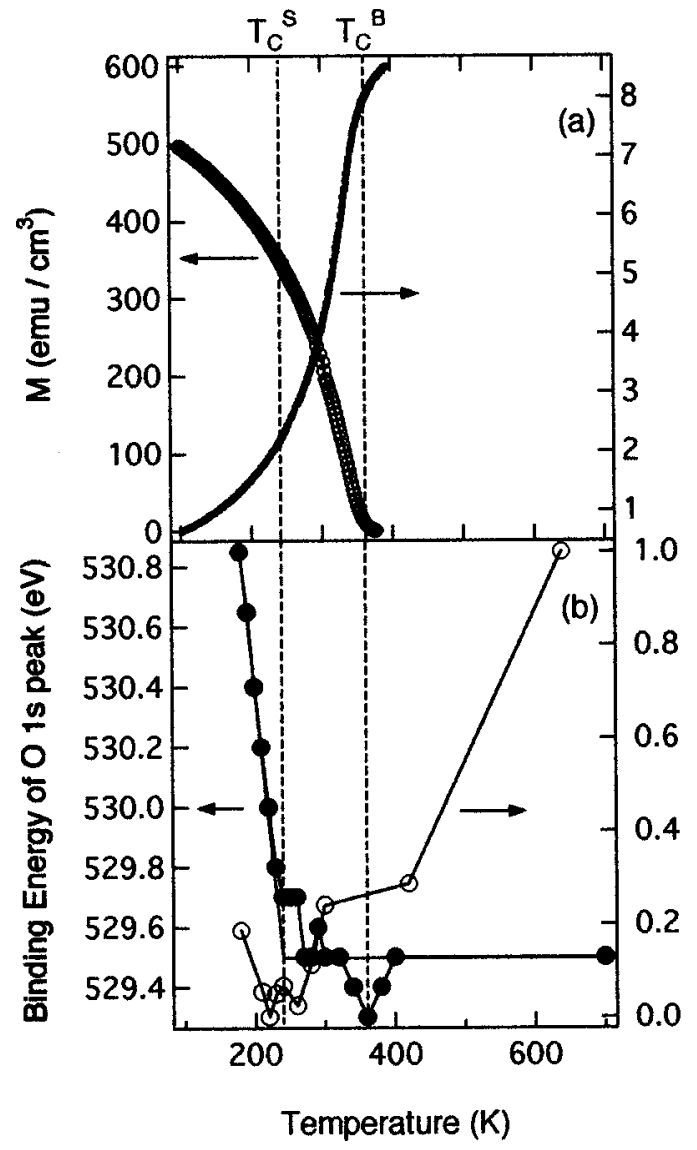

FIG. 1. Upper panel shows the bulk transition in our measurement of resistivity and bulk magnetization. Lower panel shows the binding energy of the oxygen $1 s$ peak (closed-circles) and indicators of the surface transition in the density of states, from the inverse photoemission spectra, near $E_{f}=E_{F}$ (open circles) all as functions of temperature.

as a function of temperature measured with inverse photoemission. There is no measurable density of states at the Fermi energy below $\sim 240 \mathrm{~K}$ indicating that the surface is insulating at low temperatures, consistent with the core level data. From all of this data we conclude that there is an electronic transition in the surface at $T_{C}^{S}=240 \pm 10 \mathrm{~K}$. Below this temperature the surface is an insulator, while above this temperature the surface is metallic or semimetallic.

Figure 2 displays the inverse photoemission spectra, taken at $k_{\|}=0.13 \AA^{-1}$, as a function of the sample temperature. The shaded region shows the energy segment of $\Delta E$ $= \pm 0.3 \mathrm{eV}$ which was integrated to produce the density of state data presented in Fig. 1(b). There is no measurable intensity (above background) at the Fermi energy for temperatures below $\sim 240 \mathrm{~K}$, within the energy resolution envelope of $\pm 0.3 \mathrm{eV}$. As the temperature is reduced well below $240 \mathrm{~K}$, two new, well defined, inverse photoemission features appear in the spectra at binding energies of $\sim 3$ and $\sim 4.5 \mathrm{eV}$ above the Fermi energy. The geometry of this experiment was chosen (10 deg off normal) to dramatize these two unoccupied states. These two empty states observed in the low temperature insulating phase have quite different dispersion, which can be measured by changing the angle of the incident electron beam with respect to the surface normal. The dispersion is shown in the inset. The peak in the $180 \mathrm{~K}$ spectrum labeled $L_{M}$ displays considerable dispersion with an effective mass of $\sim 0.5 \mathrm{~m}_{e}$. In contrast, the other peak in Downloaded 06 Sep 2006 to 129.93.16.206. Redistribution subject

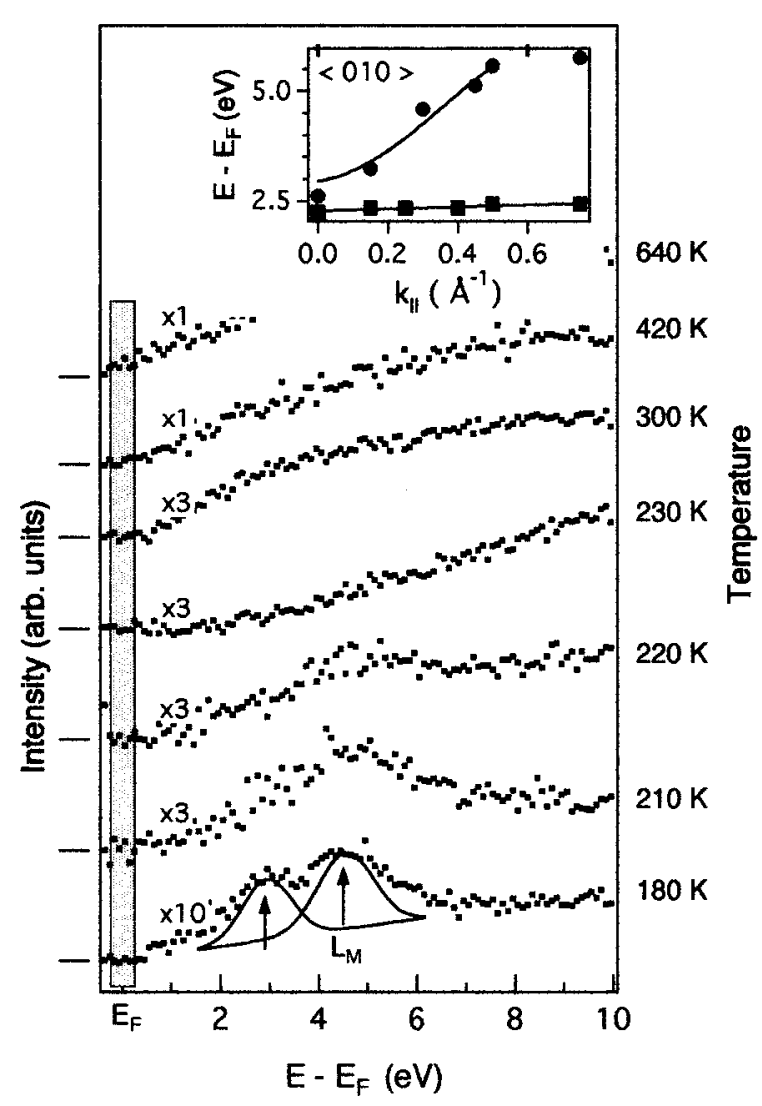

FIG. 2. Inverse photoemission spectra of $\mathrm{La}_{0.65} \mathrm{Sr}_{0.35} \mathrm{MnO}_{3}$, at an incidence angle of $10 \mathrm{deg}$ off normal, as a function of temperature. The inset shows the band dispersion of the marked states in spectrum taken at $180 \mathrm{~K}$.

the same spectrum at $\sim 3 \mathrm{eV}$ has no dispersion and its intensity varied from sample to sample and with the details of the cleaning and annealing process. We identify this peak with a localized defect state on the surface. Interestingly, both of these peaks are gone above the surface transition temperature, so they are associated with the low temperatureinsulating phase.

The quadratic light electron mass band which appears above the Fermi energy is reminiscent of quantum well states, except that, in this case, it applies to an insulating film setting on a metallic substrate. Inverse photoemission is sensitive to the states extending into the vacuum ${ }^{6}$ so it is difficult to tell if this state is an image potential state or quantum well state, i.e., primarily outside or inside the surface.

The angular dependence of the core levels' intensities can yield quantitative information about the surface composition of solids. ${ }^{7}$ If the relative concentration of $\mathrm{La}$ and $\mathrm{Sr}$ was independent of the depth into the sample the relative core level intensity would be roughly independent of the emission angle. The black squares in Fig. 3(a) show the measured intensity ratio of the $\left(\mathrm{Sr} 3 d_{5 / 2}+\operatorname{Sr} 3 d_{3 / 2}\right)$ to $\mathrm{La} 3 d_{5 / 2}$ core levels as a function of the emission angle. The ratio is not constant with the $\mathrm{Sr}$ to La ratio significantly increasing with increasing emission angle. Qualitatively this shows that the surface region is $\mathrm{Sr}$ rich. This data can be analyzed using a previously developed semiempirical method $^{2}$ to give a more quantitative picture of the degree of surface segregation. The XPS calculated intensity ratio $R_{c}(\theta)$ is defined as o AIP license or copyright, see http://apl.aip.org/apl/copyright.jsp 

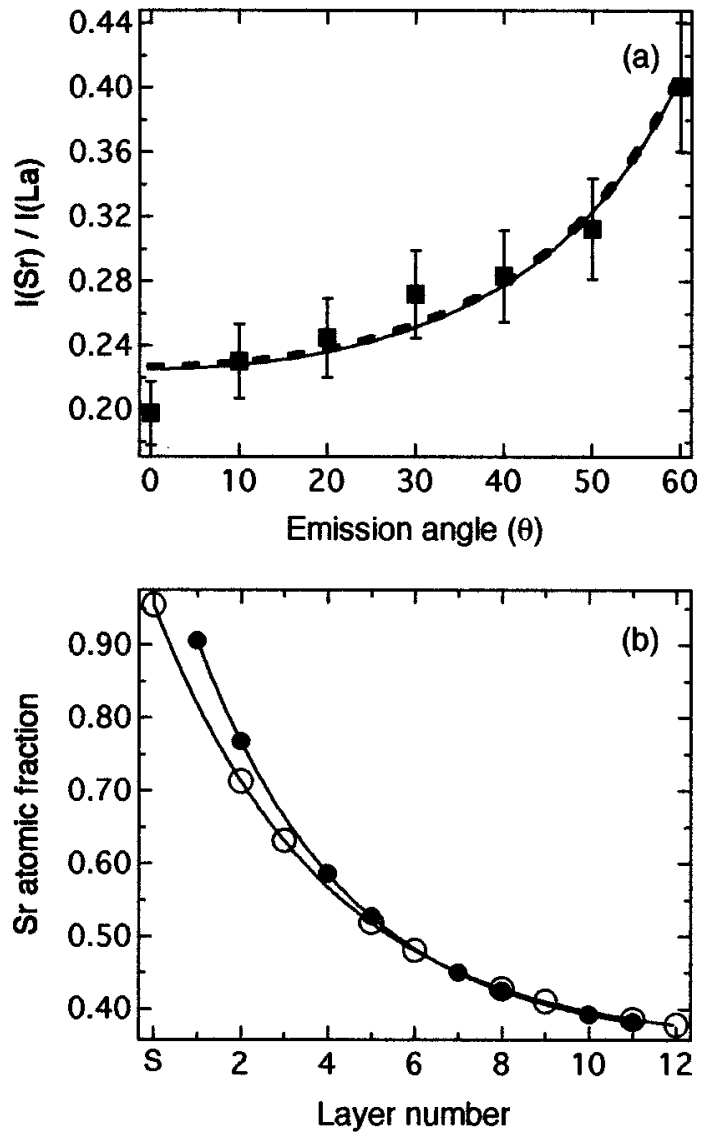

FIG. 3. Upper panel shows the relative XPS intensity of $\mathrm{Sr} 3 d$ peaks to that of La $3 d_{5 / 2}$ peak and the best fit to the data as a function of the electron emission angle. Lower panel shows the $\mathrm{Sr}$ atomic fraction as a function of the layer number (the closed circles are for the case of the $\mathrm{La} / \mathrm{SrO}$ terminal layer, whereas the open ones are for the $\mathrm{MnO}_{2}$ terminal layer).

$$
R_{c}(\theta)=\frac{\sigma_{\mathrm{Sr}}}{\sigma_{\mathrm{La}}} \frac{T_{\mathrm{Sr}}}{T_{\mathrm{La}}} \frac{\sum_{j} f_{j} \exp \left(\frac{-j d}{\sum_{\mathrm{Sr}} \cos (\theta)}\right)}{\sum_{j}\left(1-f_{j}\right) \exp \left(\frac{-j d}{\lambda_{\mathrm{La}} \cos (\theta)}\right)},
$$

where $\sigma$ is the photoionization cross section, $T$ is the transmission coefficient of the analyzer, $f_{j}$ is the atomic fraction of $\mathrm{Sr}$ at the $j$ th layer, $d$ is the interlayer distance, $\lambda$ is the inelastic mean free path of the emitted electrons, and $\theta$ is the emission angle with respect to the surface normal. The cross sections were taken from Scofield's calculations for an excitation energy of $1253.6 \mathrm{eV}(\mathrm{Mg} K \alpha)^{8}(5.29$ for $\mathrm{Sr} 3 d$ and 26.49 for La $3 d_{5 / 2}$ ). The transmission of our analyzer varies as the inverse of the kinetic energy of the emitted electrons. The mean free paths of the electrons in the material were calculated according to the scheme of Tanuma et al. ${ }^{9}(\sim 20$ $\AA$ for $\mathrm{Sr} 3 d$ and $\sim 10 \AA$ for $\mathrm{La} 3 d_{5 / 2}$ ). The $\mathrm{Sr}$ atomic fraction $f_{j}$ has been chosen by assuming an exponential segregation profile.

We found that in order to fit all intensity ratios (e.g., $\mathrm{Sr} / \mathrm{La}, \mathrm{Mn} / \mathrm{Sr}$ ) a major restructuring of the surface region need to be considered. ${ }^{10}$ Actually, the chemical composition of the surface region was found to be that of $\left(\mathrm{La}_{0.65} \mathrm{Sr}_{0.35}\right)_{2} \mathrm{MnO}_{4}\left(\mathrm{~K}_{2} \mathrm{NiF}_{4}\right.$ structure $)$ influenced by the effect of $\mathrm{Sr}$ segregation. ${ }^{10}$ Thus, the surface is a quite different material compared to the bulk.
The best fit to the data for the two possible terminations of $\mathrm{La}_{1.3} \mathrm{Sr}_{0.7} \mathrm{MnO}_{4}$ is shown in Fig. 3(a) (the solid line for $\mathrm{MnO}_{2}$ terminal layer and the dashed line for $\mathrm{La} / \mathrm{SrO}$ terminal layer). From this fitting of the measured intensity ratio, the $\mathrm{Sr}$ atomic fraction can now be plotted as a function of layer number as shown in Fig. 3(b) (the solid circles are for the case of $\mathrm{La} / \mathrm{SrO}$ terminal layer whereas the open ones are for $\mathrm{MnO}_{2}$ terminal layer). We see that there is a significant surface segregation of $\mathrm{Sr}$ to the subsurface layer. In fact, according to this fitting procedure the first layer that contains $\mathrm{Sr}$ in both terminations is almost a full $\mathrm{SrO}$ layer. This segregation profile is found to be stable over the temperature range 180 to $300 \mathrm{~K}$.

The fact that unique surface phases exist in this TMO should not come as a surprise. Interest in these materials is driven by their complex behavior, a consequence of the fact that the structural, electronic, and magnetic properties are strongly coupled. A small change in one property (e.g., structure) can produce a large change in another property (e.g., the magnetic state). ${ }^{1}$ Creation of a surface or interface is a significant change, breaking the inherent symmetry and reducing the effective dimensionality. Furthermore, all indications point to the fact that growth conditions, especially prolonged annealing, play a major role in stabilizing distinct surface phases in these materials. There is every reason to believe that the door has been opened to a new dimension in TMO phase transition associated with surfaces and interfaces. The challenge is to engineer new coupled electronic, magnetic, and structural transitions at a surface, an interfaces, or with tuned architecture. ${ }^{3}$

In closing, it is important to point out that for all of the reasons discussed above the surface of a TMO should be different from the bulk. All experimental data on these systems should be interpreted based on the premise that the surface is different electronically, structurally, and magnetically unless proven otherwise.

The primary support for this work came from the Joint Center for Atom Technologies (JRCAT) through the Atomic Technology Partnership. The effort of one author (P.A.D.) was also supported by NSF (\# DMR-98-02126) and the Center for Materials Research and Analysis (CMRA) at the University of Nebraska.

${ }^{1}$ M. Imada, A. Fujimori, and Y. Tokura, Rev. Mod. Phys. 70, 1039 (1998).

${ }^{2}$ P. A. Dowben, in Surface Segregation Phenomena, edited by P. A. Dowben and Allen Miller (CRC, Boston, MA, 1990), Chap. 6, pp. 145-168.

${ }^{3}$ M. Izumi, Y. Murakami, Y. Konishi, T. Manako, M. Kawasaki, and Y. Tokura, Phys. Rev. B 60, 1211 (1999).

${ }^{4}$ D. C. Worledge and T. H. Geballe, Appl. Phys. Lett. 76, 900 (2000).

${ }^{5}$ J. Z. Sun, D. W. Abraham, R. A. Rao, and C. B. Eom, Appl. Phys. Lett. 74, 3017 (1999).

${ }^{6}$ N. Smith, Rep. Prog. Phys. 51, 1227 (1988).

${ }^{7}$ V. I. Nefedov and O. A. Baschenko, J. Electron Spectrosc. Relat. Phenom. 47, 1 (1988).

${ }^{8}$ J. H. Scofield, J. Electron Spectrosc. Relat. Phenom. 8, 129 (1976).

${ }^{9}$ S. Tanuma, C. J. Powell, and D. R. Penn, Surf. Interface Anal. 21, 165 (1994)

${ }^{10}$ H. Dulli, P. A. Dowben, S.-H. Liou, and E. W. Plummer, Phys. Rev. B

(submitted).
AIP license or copyright, see http://apl.aip.org/apl/copyright.jsp 